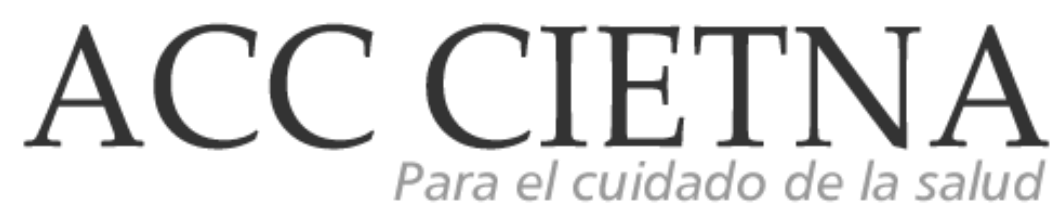

https://doi.org/10.35383/cietna.v7i1.350

\title{
Enfermería Basada en Evidencia (EBE) en el acto del cuidado enfermero: una revisión integradora
}

\section{Patiño Villalva Gladys Esther ${ }^{1}$}

INFORMACIÓN DEL ARTÍCULO
Historia del artículo:
Recibido el 01 de mayo de 2020
Aceptado el 30 de mayo de 2020

\section{Palabras clave:}

Cuidado de Enfermería

Enfermería basada en la evidencia

\begin{abstract}
RESUMEN
Objetivo: Determinar el uso de la Enfermería Basada en la Evidencia hospitalaria por los profesionales de enfermería. Marzo - julio 2019. Método: Revisión integradora de la literatura indexada, sobre la EBE y las posibilidades en relación a la aplicación de la misma en la práctica de enfermería hospitalaria, con las consideraciones de algunas barreras que no permiten su uso. La lectura crítica fue efectuada con la literatura encontrada desde 2015 a 2019 en 3 bases de datos: Bireme, Scielo, PubMed, además de libros. Resultados principales: la revisión de la literatura de 18 artículos comprobó que el 39\% fueron investigaciones de tipo cualitativo, mientras que el $39 \%$ eran cuantitativas y el $22 \%$ fueron mixtas; el predominio de las investigaciones fue en idioma español con el $61.1 \%$ y de estas el $55 \%$ fueron de investigadores españoles. El estudio permitió conocer la importancia de la evidencia científica y su aplicación en el cuidado acorde con los adelantos científicos, técnicos y humanos. Conclusión general: Las mejores evidencias disponibles en la EBE, deben ser adecuadas a las preferencias y necesidades de los usuarios y usuarias de los servicios; pero las mismas se pierden por el accionar de algunas barreras que afectan su aplicación, entre estas el distanciamiento entre el conocimiento teórico vs el práctico.
\end{abstract}

\footnotetext{
IEspecialista en Enfermería Quirúrgica. Ms. Gerencia Integral de Salud para el Desarrollo Local Universidad Técnica Particular de Loja (UTPL), Loja, Ecuador. Magister em Investigación para la Salud en la Universidad de Cuenca, Ecuador. Doctor of Philosophy, PhD with a major in Helth Sciences Atlantic International University (AIU), Hawái, Estados Unidos. Doctoranda del Programa de Doctorado en Enfermería de la Universidad Católica Santo Toribio de Mogrovejo, Chiclayo, Perú. E-mail: gladis.estherpatino@gmail.com. ORCID https://orcid.org/0000-0003-4909-3589
} 
Evidence based nursing (EBE) in the act of nursing care: an integrative review

ABSTRACT

Keywords:

Nursing Care

Evidence-Based Nursing

Hospitalary
Objective: To determine the use of evidence-based nursing on hospital by nursing professionals. March - July 2019. Method: Integrative review of the indexed literature, about EBE and the possibilities in relation to its application in hospital nursing practice, with the considerations of some barriers that do not allow its use. The critical reading was carried out with the literature found from 2015 to 2019 in 3 databases: Bireme, Scielo, PubMed, in addition to books. Main results: the review of the literature of 18 articles found that $39 \%$ were qualitative, while $39 \%$ were quantitative and $22 \%$ were mixed research; The predominance of the investigations was in Spanish with $61.1 \%$ and of these $55 \%$ were from Spanish researchers. The study allowed us to know the importance of scientific evidence and its application in care in accordance with scientific, technical and human advances. Overall conclusions: The best evidence available in EBE must be appropriate to the preferences and needs of the users of the services; but they are lost by the action of some barriers that affect their application, among them the distance between theoretical and practical knowledge.

\section{Introducción}

Entre los factores más importantes asociados a las mejoras en los niveles de atención de la salud suministrada por los profesionales de enfermería en los Hospitales la EBE, se ha transformado en la clave para mejorar la calidad del cuidado, con lo cual se están desarrollando prácticas con la mejor evidencia científica ${ }^{\text {. }}$

Para demostrar la importancia de la calidad del cuidado es preciso determinar cómo valorar al individuo y familia según sus necesidades y circunstancias; sin embargo, al solo utilizar el conocimiento obtenido en la práctica cotidiana 2,3 y no en la mejor evidencia científica existente, hace que la calidad requerida para el cuidado brindado disminuya o se pierda ${ }^{4}$. Lo anterior es una situación que conlleva establecer como un aporte la posibilidad de que la EBE se aplique para orientar el cuidado, y este evento se torna real cuando se refuerzan las voluntades de capacitación y motivación institucional para que su uso sea permanente en el personal.

De la misma forma, se espera que la EBE se transforme en un motor que motive e impulse para que otros $y$ otras profesionales de la enfermería realicen nuevos estudios, y a su vez, promuevan el uso de nuevas prácticas que posibiliten implementar la disminución de la rutina. Esto a su vez impulse, la aplicación del pensamiento crítico y la evidencia científica en el desarrollo de su praxis clínica ${ }^{5}$.

\section{Metodología}

Para este estudio se aplicó la metodología de Revisión Integradora de la literatura ${ }^{6}$, lo que se buscaba es comprender la EBE mediante un proceso de sistematización y análisis de todos los artículos y libros. El estudio se realizó en seis etapas: 
Primero: pregunta orientadora ¿qué encontramos en la literatura indexada sobre las posibilidades y barreras de la aplicación de la EBE en la práctica de enfermería hospitalaria?

Segundo: Criterios de inclusión: que el artículo sea original, el tiempo de búsqueda se encuentre situado entre los años 2015 al 2019; se consideraron las bases de datos: Bireme, PubMed y Scielo; y dentro de éstas, portales de revistas como: Wiley, Elsevier, Redalyc, Medigraphic, BMC Public Health y Dialnet; además de 3 libros como fuente primaria que orientaron del conocimiento sobre el tema.

Para la fórmula de búsqueda se utilizaron descriptores en ciencias de la salud (DeCS) unidos por los operadores boleanos AND y OR, de la siguiente forma: (Nurses'[All Fields] AND ("evidence-based practice"[MeSH Terms] OR ("evidence-based"[All Fields] AND "practice"[All Fields]) OR "evidence-based practice"[All Fields] OR ("evidence"[All Fields] AND "based"[All Fields] AND "practice"[All Fields]) OR "evidence based practice"[All Fields])) AND ("loattrfree full text"[sb] AND "2015/06/24"[PDat]: "2019/06/22"[PDat]).

Los descriptores de la búsqueda se asociaron a otros términos como: "calidad del cuidado" para visibilizar la utilidad práctica de la EBE en relación a la calidad del cuidado; "posibilidades" para buscar si es posible la aplicación de la EBE y "barreras" como necesidad de conocer si existen razones para la no aplicación de la EBE a nivel hospitalario. Los idiomas que se incluyeron fueron: español e inglés.

Tercera: presentar las estadísticas de los estudios seleccionados en tablas, se consideró para su presentación las palabras claves utilizadas, el objetivo del estudio además de las posibilidades y barreras para su implementación a nivel hospitalario.
Cuarta: análisis crítico de los hallazgos buscando similitudes y diferencias de los temas relativos a la EBE.

Quinta: la interpretación de los resultados, la misma que fue ejecutada bajo las categorías de posibilidades y barreras para aplicación de la EBE en el área hospitalaria.

Sexta: Desarrollo y presentación del informe final, aquí se mostró la evidencia de toda la información obtenida.

\section{Resultados y discusión}

Se desarrolló la revisión por títulos y resúmenes para verificar su aporte al tema, aquí se seleccionaron un total de 220 artículos; después de realizar una nueva lectura crítica quedaron incluidos 24 artículos, y, de estos se retiraron 6 por ser estudios de revisión integrativa (artículos de revisión secundaria), al final quedaron 18 artículos. 
Cuadro $N^{\circ} 1$. ARTíCULOS DE BASES DE DATOS

OBTENIDOS EN LOS MESES ABRIL-JULIO DE 2019

\begin{tabular}{|c|c|c|c|c|}
\hline Base de datos & BVS & PubMed & Scielo & Repositorios \\
\hline Descriptores & $\begin{array}{l}\text { Cuidado de } \\
\text { Enfermería (Q65.030), } \\
\text { Enfermería basado en } \\
\text { evidencia } \\
\text { (H02.249.875), } \\
\text { hospitalarios } \\
\text { (E02.760.611) }\end{array}$ & $\begin{array}{l}\text { Cuidado de } \\
\text { Enfermería, } \\
\text { Enfermería } \\
\text { basado en } \\
\text { evidencia, } \\
\text { hospitalarios. }\end{array}$ & $\begin{array}{l}\text { Cuidado de } \\
\text { Enfermería, } \\
\text { Enfermería } \\
\text { basado en } \\
\text { evidencia, } \\
\text { hospitalarios. }\end{array}$ & $\begin{array}{l}\text { Tesis } \\
\text { Cuidado de } \\
\text { Enfermería, } \\
\text { Enfermería basado } \\
\text { en evidencia, } \\
\text { hospitalarios. }\end{array}$ \\
\hline $\begin{array}{l}\text { Artículos } \\
\text { encontrados }\end{array}$ & 10915 & 620 & 169 & 5 \\
\hline $\begin{array}{l}\text { Artículos } \\
\text { seleccionados de } \\
\text { acuerdo con los } \\
\text { criterios de inclusión }\end{array}$ & 84 & 119 & 17 & 3 \\
\hline 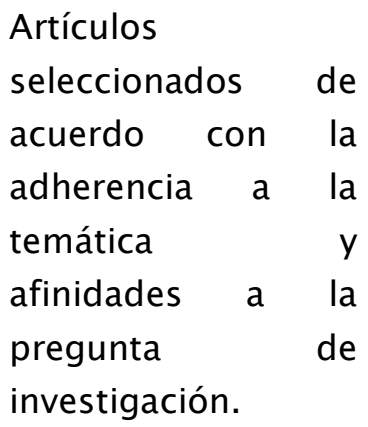 & 6 & 11 & 7 & 3 \\
\hline \multicolumn{3}{|c|}{ Artículos retirados luego de nueva revisión } & 6 & 0 \\
\hline \multicolumn{3}{|c|}{ Muestra } & 18 & 3 \\
\hline \multicolumn{3}{|c|}{ Artículos repetidos entre las bases } & 12 & 0 \\
\hline
\end{tabular}

Fuente: Base de datos obtenidos de los estudios

Realizado por: la Investigadora

De la muestra seleccionada el 39\% corresponde a estudios de tipo cualitativo: fenomenológicas, hermenéuticas y heurísticas; estos estudios, utilizaron técnicas como: trabajos en grupo, entrevistas a profesionales, jefes de enfermería y especialistas, las investigaciones profundizaron sobre el tema de calidad, posibilidades y barreras sobre la aplicación de la EBE a nivel hospitalario e institucional.

Además, en la selección hubo el $39 \%$ de estudios de tipo cuantitativo, los cuales han demostrado estadísticamente el problema de las barreras, así como de las posibilidades encontradas para la aplicación de la EBE a nivel hospitalario, estos estudios en su mayoría tratan sobre la aplicación de los cuestionarios: (EBPQ) y Nurs-ing Work Index (NWI) aplicados a profesionales antiguos y de nuevo ingreso que laboran en hospitales y a nivel de APS. La finalidad de estos cuestionarios persiguió obtener información sobre 5 dimensiones: 1) participación de la enfermera en asuntos del hospital; 2) fundamento del enfermero sobre la calidad de los cuidados; 3) capacidad de liderazgo y apoyo a las enfermeras por parte de los gestores enfermeros; 4) plantilla y adecuación de los recursos humanos; 5) relaciones entre médicos y enfermeras. Además, hubo 4 artículos, con 3 tesis encontradas en repositorios de universidades, que fueron 
investigaciones desarrolladas utilizando tanto la investigación mixta.

En cuanto al idioma utilizado el $61.1 \%$ optó por el español, y el 38.9\% por el inglés; de este último, 4 de los 7 artículos revisados fueron investigaciones de tipo cuantitativo.

La EBE en sus inicios:

La Práctica Basada en la Evidencia (PBE) o (EBE) Enfermería Basada en la Evidencia tiene sus inicios en la medicina basada en la evidencia, iniciando su desarrollo en Canadá, con base en la epidemiología clínica, debido a que el grupo de Sackett, a fines de los años 70 buscaba incorporar la investigación clínica en la toma de decisiones para los pacientes. Otro grupo canadiense liderado por Guyatt en los años 90 promovió la utilización de investigaciones con base matemático - estadístico como evidencia?

Otra de las razones por las que se motivó el uso de la EBE, fue porque se produjo un desfase entre la aparición de libros y la literatura científica periódica, la variabilidad en la atención clínica en pacientes con una misma enfermedad, los distanciamientos entre los avances científicos y la práctica asistencial, en esta última, se dio importancia al manejo del conocimiento resultado de la experiencia propia o del profesional más antiguo en el servicio; por lo que, la laguna existente entre investigación vs práctica facilitaron el nacimiento ${ }^{1}$ de este nuevo paradigma "La Enfermería Basada en la evidencia”.

Lo importante para la comunidad de enfermería siempre fue y será la demanda de los conocimientos que aportan las investigaciones que emergen del análisis y la trascendencia de los proyectos y su aplicabilidad en la profesión( ${ }^{(8)}$ según las características de los contextos en que se da en cada país tanto en la formación de pre y postgrado como en la práctica y aplicación del cuidado como objeto de la profesión.
Fases de la Enfermería Basada en la Evidencia para su aplicación:

Al pensar en el desarrollo de la investigación, impele a tener muy claro desde que visión de la ciencia será desarrollado el estudio si, desde la perspectiva cualitativa, cuantitativa o mixta, todos los métodos son muy importantes $y$ depende que es lo que se quiere demostrar para elegir la más relevante, ya que pensar en ¿para qué? ¿por qué? ¿para quién? Corresponde encontrar una respuesta ya sea en situaciones que nos ayuden a:

1) Comprender el porqué de la utilidad de una intervención.

2) Identificar cuáles son los tópicos adecuados para la investigación cualitativa.

3) Explicar resultados inesperados de la investigación cuantitativa

4) Generar hipótesis experimentales ${ }^{9}$

Figura $\mathrm{N}^{\circ} 01$. Complementariedad existente entre la investigación cuantitativa y cualitativa para generar tipos de conocimiento

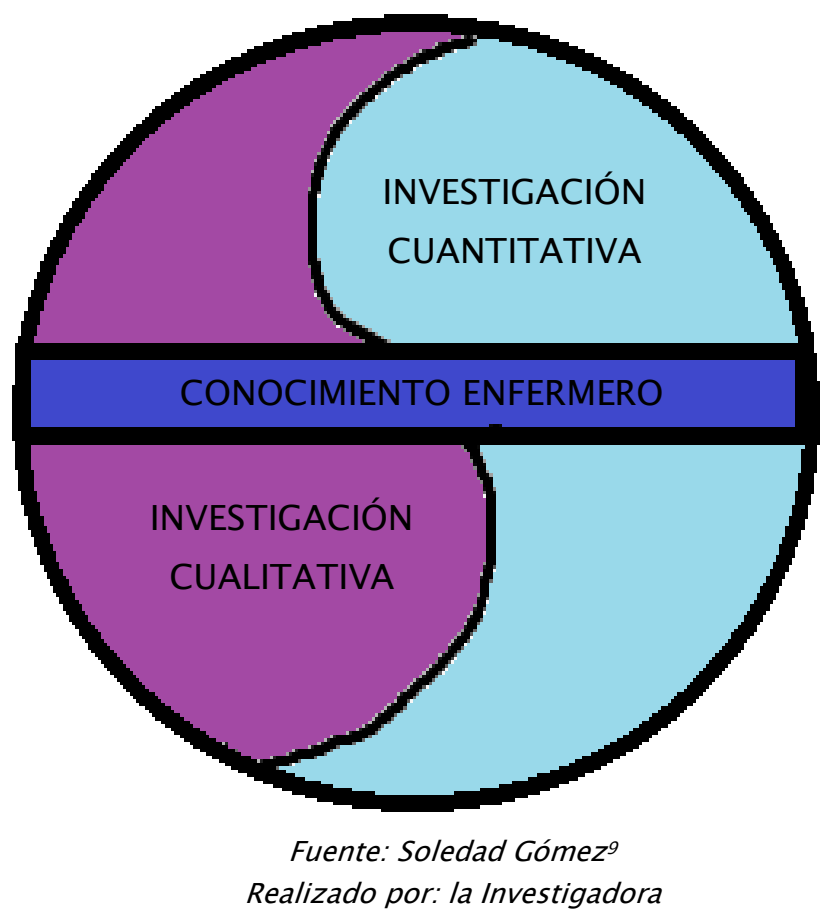

Cuando se realiza el cuidado enfermero surgen muchas preguntas y dudas, las mismas que van a orientar la búsqueda del conocimiento, y/o en caso que en la EBE no exista una respuesta para 
dicho cuidado, ello plantea la posibilidad de realizar una investigación original y de allí nace el aporte al crecimiento y fortalecimiento de la EBE y de la profesión de enfermería ${ }^{10}$.

Figura 2. Una visión de la EBE como nuevo paradigma en enfermería

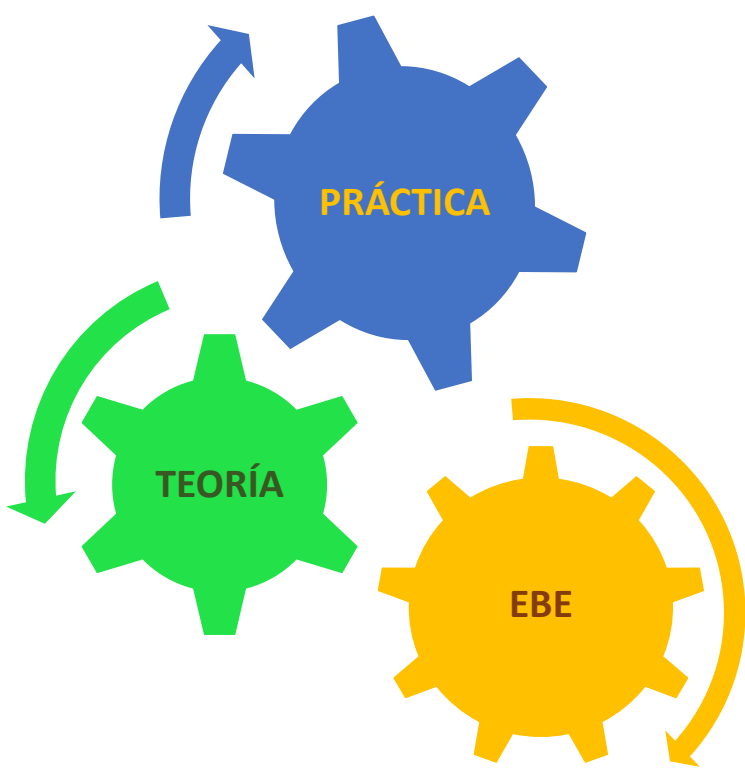

Realizado por: Elaboración propia. Aporte de la Investigadora.

La teoría enriquece la EBE y la EBE fortalece la práctica enfermero, por lo tanto, los tres en conjunto aportan al desarrollo sostenido de cada uno, denominándose PBE.

En este momento vale una reflexión, que si bien la investigación cuantitativa nos provee de un valioso conocimiento estadístico, objetivo y de razón que facilita la comprensión de la realidad existente en ese momento, por otro lado, el conocimiento que nos brinda la investigación cualitativa ayuda al personal de enfermería a comprender la problemática profunda espiritual y psicológica por la que está cursando el paciente y posibilita desarrollar un trabajo más personalizado y específico para dicha persona, familia o comunidad.

Para conseguir conocimientos y habilidades por medio de la aplicación del proceso de la EBE en la PBE (práctica basada en la evidencia) ${ }^{5}$ es imperioso ofrecer a las enfermeras los instrumentos necesarios para posesionarse de sus prácticas, mejorar la atención de salud y el cuidado del paciente.

La EBE en la realidad de la profesión de enfermería:

La EBE es considerada como"1: "la utilización consciente, explícita y juiciosa (crítica) de la información derivada de la teoría; la información basada en investigación en la toma de decisiones para el cuidado que se da a individuos o grupos de pacientes en consideración con las necesidades y preferencias individuales".

Desde las variaciones en los patrones de provisión de cuidados sobre la morbilidad del paciente hospitalizado, se suman cambios en los paradigmas vinculados con el pensamiento enfermero.

Desde hace unos años, el movimiento denominado ${ }^{12}$ Enfermería Basada en la Evidencia (EBE), recomienda que todas las acciones realizadas en la práctica clínica tengan como respaldo una base científica. Solo con la selección de estas prácticas se lograría mediante la revisión crítica de las investigaciones basadas en un alto rigor metodológico, mejorar la calidad del cuidado enfermero.

Fases de la Enfermería Basada en la Evidencia para su aplicación: 
Figura 3: Fases de la EBE

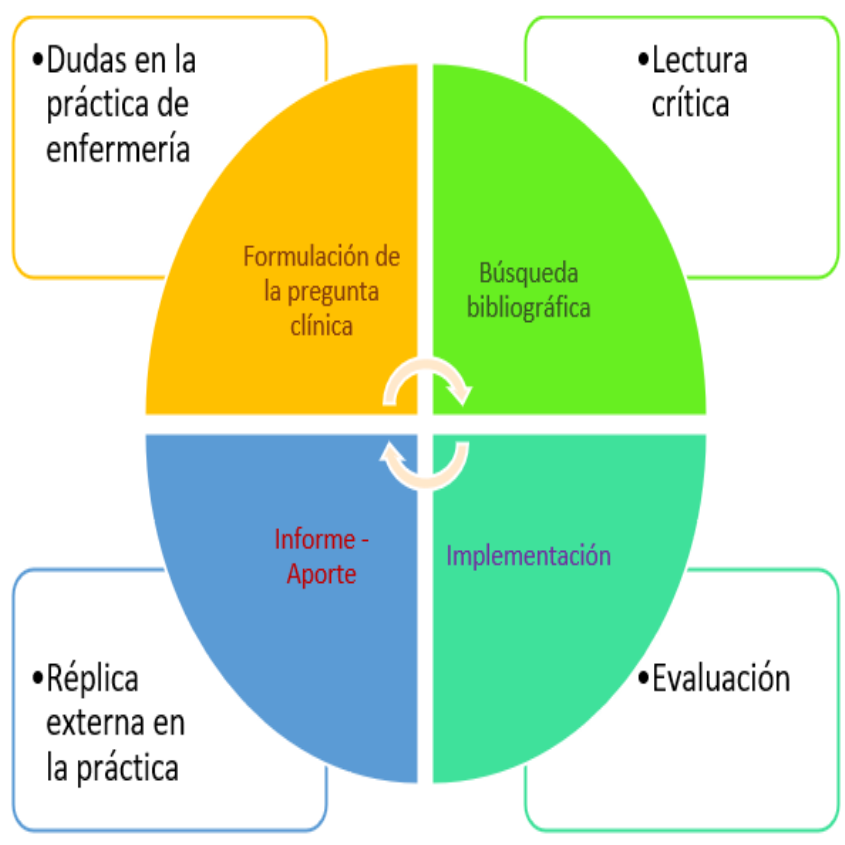

Realizado por: la Investigadora. Elaboración propia.

Las capacidades que desarrolla la EBE según Grove $^{13}$ al desarrollar el cuidado enfermero, permite que el/la profesional utilicen el conocimiento existente en la EBE o a su vez formule la pregunta de investigación necesaria para realizarla. Luego al momento que realiza la búsqueda bibliográfica es muy importante no solo que se efectúe sobre la lectura de artículos científicos y libros, sino que, esa lectura sea crítica con el fin de que ella aporte a la investigación, la evaluación y al nuevo conocimiento.

Al final, la réplica del cuidado produce la validez externa necesaria para generalizar por medio del informe el conocimiento y la técnica para toda la Comunidad Enfermera.

Los elementos clave para la aplicación de la EBE según los investigadores, son los tutores EBE que deben poseer las instituciones y las asociaciones. Ellos son profesionales valiosos para el ambiente tanto académico como clínico; asimismo, la investigación, el tiempo, los recursos y el apoyo administrativo son fundamentales para direccionar la asociación de la evidencia a la práctica enfermera.
Barreras para la implementación de la EBE en los hospitales:

A pesar que, la EBE ha aportado profundamente ${ }^{14}$ como una estrategia relevante para el desarrollo y mejora de la calidad del cuidado. En el 2006 se demostró que no se podía hablar aún de la Enfermería como una profesión basada en la investigación, porque, aunque los profesionales consideraban como muy positiva la investigación, resultaba por distintas razones para ellos muy dificultoso aplicar los resultados encontrados.

Lo anterior se problematiza aún más, porque el trabajo que se ha realizado a nivel del sistema de salud para la implementación de la EBE en Hospitalización y APS, se ve afectada según indican algunos investigadores 15,16,17,18 ante algunas barreras ocasionadas por la falta de motivación que reciben los profesionales por parte de los mismos administradores a nivel institucional para la implementación de la PBE.

Lo anterior, ha sido percibido por las enfermeras que fueron parte del estudio como barreras que además están relacionadas con situaciones personales: la falta de conocimientos, no actualizan los conocimientos y refieren resistencia a modificar sus prácticas. A nivel institucional: reciben falta de apoyo, poca motivación y no se facilita las bases de datos que necesitan los profesionales por parte de la institución y la propia coordinación de enfermería para la búsqueda. Por estas razones indican que existe una gran distancia entre el conocimiento teórico vs el práctico evidenciado en los resultados de cuidados obtenidos.

De esta manera, en la actualidad el desarrollo del plan de cuidados se produce sobre la base de la experiencia cotidiana, esto ha permitido que la calidad del cuidado vaya disminuyendo, si abrimos el accionar a una nueva visión de un paradigma más motivador ${ }^{15}$, interactivo y de aporte científico permitirá que enfermería en cualquier contexto se fortalezca $y$ en 
consecuencia también lo haga el "cuidado enfermero".

La actualización continua de los conocimientos junto con el acelerado desarrollo científico y tecnológico abre las puertas del conocimiento lo mismo que obliga a que todos los profesionales de la salud y en especial los de enfermería sientan la necesidad de prepararse permanentemente ${ }^{19}$, razón por la cual los enfermeros/as deban empoderarse de dicho conocimiento y practicar la EBE para la toma de decisiones. Además, es importante que las mismas contribuyan a elevar la calidad de los cuidados aplicados al paciente que lo necesita de forma técnica, científica, personalizada y conjuntamente con ello allanar el camino para la aplicación adecuada de la EBE.

Existe abundante literatura, pero ellas en su mayoría no cumplen con los criterios de inclusión por lo que el número de artículos fueron disminuidos, sin embargo, todos los artículos escogidos han posibilitado el entendimiento más claro sobre el fenómeno estudiado "la EBE"

El fortalecimiento de la enfermería como ciencia en construcción ${ }^{10}$ requiere de utilizar como estrategia la formación de redes colaborativas y tutores EBE en las instituciones que brindan atención de salud para alcanzar el desarrollo de conocimientos científicos propios de la profesión, su divulgación fortalecerá la EBE y la aplicación práctica por los profesionales que brindan el cuidado², lo mejorará en los pacientes que son atendidos en los hospitales.

Lo importante de la EBE es que, es un conocimiento obtenido por la investigación, la misma ha sido probada y comprobada en base a la aplicación de un método científico y toda la información o resultados obtenidos se ven evidenciados en los artículos científicos que son editados por bases de datos, de allí nace la importancia de que los hospitales y los profesionales tomen conciencia de la importancia de su implementación ${ }^{13,4}$ al momento de aplicar los cuidados o de realizar protocolos de atención que luego serán estandarizados para el cuidado administrado a los pacientes en todo el hospital.

Cabe destacar, que enfermería necesita de afianzarse de nuevos conocimientos y prácticas que posibiliten transformase en profesionales propositivos y proactivos que permitan que la profesión en su teoría y práctica se enriquezca20, y que los pacientes quienes reciben la atención sean los mayores beneficiarios del cuidado.

En los hospitales según estudios realizados por algunos investigadores se encuentra que la institución hospitalaria es la que más barreras pone para la aplicación de la $E_{B E}^{21}$, incluso según los investigadores, algunas de las profesionales entrevistadas indican que sienten que la institución no motiva en el personal a la investigación como un pilar fundamental de la práctica; de acuerdo con sus respuestas, este sería uno de los puntales por los que no se les facilitaría el mantener o acoger a la EBE para el desarrollo de la práctica clínica ${ }^{18}$.

Lo importante es obtener que otros servicios $u$ otros hospitales 22 desarrollen trabajos que posibiliten la aplicación de la EBE en el cuidado de enfermería y que por ende se coadyuve para que se rompan barreras y se posibilite el uso y aplicación de la abundante información e investigación encontrada en la EBE.

Algo muy importante que consideran los investigadores es que, existe el conocimiento tácito $^{3}$ nacido desde la experiencia de trabajo cotidiano, éste de alguna forma se transforma en una barrera para que la EBE no pueda implementarse en el cuidado enfermero; pues, si ese conocimiento tácito adquirido por medio de la experiencia se une a la transferencia del conocimiento entre pares se transforma en un conocimiento que conjuntamente con la intuición se hace más fuertemente utilizado al momento de administrar los cuidados, dándoles a los profesionales una falsa seguridad del 
conocimiento y de ahí su negación de utilizar la EBE.

\section{Conclusión}

Las mejores evidencias disponibles en la EBE, deben ser adecuadas a las preferencias $y$ necesidades de los usuarios y usuarias de los servicios; pero las mismas se pierden por el accionar de algunas barreras que afectan su aplicación, entre estas el distanciamiento entre el conocimiento teórico vs el práctico.

La EBE es el mejor proceso que posibilita incrementar la calidad de atención de los pacientes en el cuidado enfermero y deben ser aplicadas para mejorar la calidad y la relación costo - beneficio - efectividad de las acciones de salud prestadas por enfermería al paciente hospitalizado.

La resistencia a la utilización de la EBE como la mejor evidencia disponible, según los investigadores las causas son el desconocimiento o una dificultad en el análisis crítico de la literatura, falta de investigaciones en los hospitales y resistencia a modificar prácticas las mismas que son incorporadas por el conocimiento tácito o conocimiento producto de la práctica cotidiana. Los resultados obtenidos orientan la importancia de desarrollar un estudio sobre la realidad que envuelve el ambiente de la profesión y del cuidado en relación a la aplicación de la EBE y la práctica cotidiana en el cuidado de los pacientes atendidos por la/el profesional de enfermería en los hospitales.

\section{Bibliografía}

1. Medrano-Mariscal J. Acercamiento al descriptor Enfermería Basada en la Evidencia desde una perspectiva compleja. Rev Enfermería del Inst Mex del Seguro Soc [Internet]. 2017 May 15 [cited 2019 Jun 23];25(2):145-50. Available from: https://www.medigraphic.com/cgi- $\mathrm{bin} / \mathrm{new} /$ resumen.cgi?IDARTICULO $=72008 \&$ id $2=$

2. De Pedro-Gómez JE. Of evidence-based practice to advanced nursing practice. Rev Mex Enfermería Cardiológica [Internet]. 2015 [cited 2019 Jul 1];23(2):62-5. Available from: www.medigraphic.org.mxhttp://www.medig raphic.com/enfermeriacardiologicawww.me digraphic.org. $m x$

3. Pérez-Fuillerat N, Solano-Ruiz MC, Amezcua M. Conocimiento tácito: características en la práctica enfermera. Gac Sanit [Internet]. 2019 Mar [cited 2020 May 6];33(2):191-6. Available

from: https://linkinghub.elsevier.com/retrieve/pii /S0213911117303114

4. Rye M, Friborg O, Skre I. Attitudes of mental health providers towards adoption of evidence-based interventions: relationship to workplace, staff roles and social and psychological factors at work. BMC Health Serv Res [Internet]. 2019 Dec 8 [cited 2019 Jul 10];19(1):110-22. Available from: https://bmchealthservres.biomedcentral.co m/articles/10.1186/s12913-019-3933-4

5. Falconí Morales, Carmen; Brito Santacruz, Carmita J; Vercovitch I. Integración de la enseñanza de la práctica de enfermería basada en la evidencia científica. Aquichan [Internet]. 2015 [cited 2019 Jul 18];15(4):114. Available from: http:/ / www.redalyc.org/pdf/741/74142431 009.pdf

6. Carvajal EY, Sánchez Herrera B. Los modelos de enfermería aplicados en la práctica clínica: revisión integrativa. Arch Med [Internet]. 2018 Jun 20 [cited 2019 Aug 3];18(1):86-96. Available from: http://revistasum.umanizales.edu.co/ojs/in dex.php/archivosmedicina/article/view/ 170 1

7. Orellana Yañez A, Paravic Klijn T. Enfermería basada en evidencia: barreras y estrategias para su implementación. Cienc y enfermería [Internet]. 2007 Jun [cited 2019 Apr 6];13(1): 17-24. Available from: 
http:/ / www.scielo.cl/scielo.php?script $=$ sci $_{-}$ arttext\&pid $=$ S0717-

$95532007000100003 \& \operatorname{lng}=$ en\&nrm $=$ iso\&tl $\mathrm{ng}=\mathrm{en}$

8. Medina Gonzáles, Inarvis; Tafur Castillo, Janery del Socorro; Vigil de Hernández, María Alicia; Hernández García R. La internacionalización y el desarrollo de la enfermería como ciencia desde los intercambios científicos. ems.sld.cu [Internet]. 2018 [cited 2019 Mar 30]; 32(4): 268-92. Available from: http://www.ems.sld.cu/index.php/ems/arti cle/view/1602

9. Gomez S. Implementación de la EBE en el Cuidado del Paciente Crítico. Trabajo final integrativo. [Internet]. Universidad Nacional de Córdova; 2016 [cited 2019 Jul 18]. Available from: http://lildbi.fcm.unc.edu.ar/lildbi/tesis/go mez-SOLEDAD.pdf

10. Mónica Motta Lino1, Vânia Marli Schubert Backes1, María Arminda da Silva Mendes Carneiro da Costa2, María Manuela Ferreira Pereira da Silva Martins2 MML. Aspectos epistemológicos de la nueva investigación en Enfermería: colaborativa, transdisciplinaria y translacional. Index de Enfermería [Internet]. 2017 [cited 2019 Jul 13]; 26(1-2): 118-22. Available from: http://scielo.isciii.es/scielo.php?pid=S1 132

$12962017000100027 \&$ script $=$ sci_arttext $\&$ tl $\mathrm{ng}=\mathrm{en}$

11. Molina Mula, Jesús; Vaca Auz, Janeth; Muñoz Navarro, Paulina; Cabascango Cabascango, Katty; Cabascango Cabascango C. Gestión de la calidad de los cuidados de enfermería hospitalaria basada en la evidencia científica. SciELO Espana [Internet]. 2016 [cited 2019 May 10]; 25(3): 151-5. Available from: http://scielo.isciii.es/scielo.php?script $=$ sci_ arttext\&pid =S1 132-12962016000200006

12. Hine-Sanabria $A$, Mendoza-Monsalve $A$, Rojas LZ, Roa-Díaz ZM, Hine-Sanabria A, Mendoza-Monsalve $A$, et al. Conocimiento e investigación sobre la autonomía profesional en enfermería. Revisión narrativa. Enfermería Univ [Internet]. 2018 Aug 10 [cited 2019 Jun 22];15(2):212-21. Available from: http://revistaenfermeria.unam.mx:80/ojs/index.php/enf ermeriauniversitaria/article/view/501

13. Grove SK, Gray J, Burns N. Investigación en enfermería: desarrollo de la práctica enfermera basada en la evidencia [Internet]. 6ta ed. Barcelona: Elsevier; 2016 [cited 2019 Apr 9]. 596 p. Available from: http://paltex.paho.org/Publication?publicati onld $=86 f 3 a 36 b-68 d 6-4 e a 2-8826-$ $81 \mathrm{~d} 51 \mathrm{~d} 06993 \mathrm{~b}$

14. Rodríguez Campo V, Paravic Klijn T. Enfermería basada en la evidencia y gestión del cuidado. Enfermería Glob [Internet]. 2011 Oct 6 [cited 2019 Apr 6]; 10(4). Available from: http://revistas.um.es/eglobal/article/view/ 137481

15. Susana González Torrente. Factores que influyen en la incorporación de la evidencia científica a la práctica diaria de las enfermeras de Atención Primaria [Internet]. Universitat de les Illes Balears; 2014 [cited 2019 Jul 13]. Available from: https://www.infermeriabalear.com/registro. php?idgrupo $=20160115121606 \&$ lan $=1$

16. Cidoncha-Moreno $M$, Ruíz de AlegríaFernandez, A; de Retana B. Barriers to the implementation of research perceived by nurses from Osakidetza. Enfermería Clínica (English Ed. 2017; 27(5): 286-93.

17. Llauradó-Serra M, Güell-Baró R, CastaneraDuro A, Sandalinas I, Argilaga E, Fortes-del Valle $\mathrm{ML}$, et al. Barreras y motivaciones de los profesionales de enfermería para la utilización de la investigación en Unidades de Cuidados Intensivos y en el Servicio de Emergencias Médicas. Enfermería Intensiva. 2016; 27(4):146-54.

18. Nkrumah I, Atuhaire C, Priebe G, Cumber SN. Barriers for nurses' participation in and utilisation of clinical research in three 
hospitals within the Kumasi Metropolis, Ghana. Pan Afr Med J [Internet]. 2018 [cited 2019 Jul 10]; 30: 24. Available from: http://www.ncbi.nlm.nih.gov/pubmed/302 14657

19. Leyla Alegría1, Víctor Pedrero2, Irene Fuentes3, María de los Ángeles Sanhueza4, María Toro4 PD, Vixsa Medo5, Daniela Santander6 $\mathrm{Cl}$. Documento de Consenso "Una aproximación a la Práctica de Enfermería Basada en la Evidencia en Unidad de Paciente Crítico." medicina-intensiva.cl [Internet]. 2016 [cited 2019 Jun 20]; 31 (3): 149-6. Available from: https://www.medicinaintensiva.cl/revistaweb/revistas/indice/201 $6 \_3 / \mathrm{pdf} / 2 . \mathrm{pdf}$

20. Cheng, L; Broome, ME; Feng S. Echar raíces: una teoría fundamentada sobre la implementación de la enfermería basada en la evidencia en China. dialnet.unirioja.es [Internet]. 2018 [cited 2019 May 10]; 65(2): 291-300. Available from: https://dialnet.unirioja.es/servlet/articulo?c odigo $=6558741$

21. Castro M, Simian D. LA ENFERMERÍA Y LA INVESTIGACIÓN. Rev Médica Clínica Las Condes [Internet]. 2018; 29(3): 301-10. Available from: https://www.sciencedirect.com/science/arti cle/pii/S0716864018300531

22. González-Torrente S, Pericas-Beltrán J, Bennasar-Veny $M$, Adrover-Barceló $R$, Morales-Asencio JM, De Pedro-Gómez J. Perception of evidence-based practice and the professional environment of Primary Health Care nurses in the Spanish context: a cross-sectional study. BMC Heal Serv Res [Internet]. 2012 [cited 2019 May 9]; 12(227): 1-9. Available from: http:/ / www.biomedcentral.com/1472$6963 / 12 / 227$ 